\title{
Right-Versus Left-Subclavian Central Venous Port for Patients with Malignant Tumor: A Retrospective Comparison of Adverse Events and Imaging Findings
}

\author{
1) Department of Diagnostic Radiology, National Cancer Center Hospital, Tokyo, Japan
}

Miyuki Sone ${ }^{1)}$, Yasuaki Arai ${ }^{1)}$, Shunsuke Sugawara ${ }^{1)}$, Kohji Tomita $^{1)}$

\begin{abstract}
Purpose: The optimal side for placement of a central venous port (CVP) remains unclear. The authors aimed to compare adverse events (AEs) and imaging findings according to CVP placement via the right and left subclavian vein (SCV) in patients with malignant tumor(s).

Material and Methods: A total of 254 consecutive adult patients, who underwent CVP placement between January and August 2012, were included. Percutaneous ultrasound-guided access was attempted in the right SCV in 134 (59 men, 75 women; median age 61 years) and left SCV in 120 (58 men, 62 women; median age 61 years) patients. The primary tumor was colorectal (right, 17/ left, 71); upper gastrointestinal (33/7); bone and soft tissue (21/9); and others (63/33). A retrospective evaluation of technical success, AEs, and computed tomography findings was performed.

Results: Technical success was achieved in $99 \%$ of patients in both groups. Median procedure time was 30 min in both groups. There were no statistically significant differences in intraprocedural AEs. In postprocedural AEs during a median follow-up of 347 (right)/530 (left) days, CVP placement in the left SCV was associated with a significantly higher rate of catheter occlusion (right, 0\%; left, 5.8\%). Both symptomatic venous thrombosis (right, 0.7\%; left, 4.2\%) and image-confirmed venous thrombosis (right, 4.8\%; left, 21.8\%) occurred more frequently in the left-CVP group.

Conclusion: Left-CVP was associated with significantly higher rates of late AEs and venous thrombosis. Despite the inherent limitations of the retrospective study design and small sample size, re-evaluation of leftCVP may be required in patients with malignant tumor(s).
\end{abstract}

Key words: central venous port, venous access, oncologic patients

(Interventional Radiology 2018; 3: 121-125)

\section{Introduction}

Anatomically, the left subclavian vein (SCV) runs a longer course toward the superior vena cava than the right SCV because of the right side position of the vena cava in the mediastinum and circumvention of the aortic arch dorsal to the left SCV $[1,2]$. However, the clinical significance of differences between central venous port (CVP) insertion via the right or left SCV has not yet been fully evaluated; thus, the optimal side for placement of a CVP remains unclear. We aimed to compare adverse events (AEs) and imaging findings according to CVP placement via the right and left $\mathrm{SCV}$ in oncological patients.

\section{Material and Methods}

\section{Study design}

A retrospective comparative study involving review of 
Table 1. Characteristics of Patients

\begin{tabular}{lllll}
\hline Characteristic & & $\begin{array}{l}\text { Right } \\
(\mathrm{N}=134)\end{array}$ & $\begin{array}{l}\text { Left } \\
(\mathrm{N}=120)\end{array}$ & $P$ \\
\hline Age (years) & Median & 58 & 59 & .340 \\
& Range & $23-81$ & $20-85$ & \\
Gender & Male / female & $59 / 75$ & $58 / 62$ & .534 \\
Type of cancer & Colorectal & $17(13 \%)$ & $71(59 \%)$ & \\
& Upper GI & $33(25 \%)$ & $7(6 \%)$ & \\
& Pancreas & $9(7 \%)$ & $6(5 \%)$ & \\
& Musculoskeletal & $21(16 \%)$ & $9(8 \%)$ & .022 \\
& Breast & $13(9 \%)$ & $6(5 \%)$ & \\
& Female pelvis & $14(10 \%)$ & $5(4 \%)$ & \\
& Others & $27(20 \%)$ & $16(13 \%)$ & \\
Purpose of CVP & Chemotherapy & $76(57 \%)$ & $97(81 \%)$ & $<.0001$ \\
& Palliative & $58(43 \%)$ & $23(19 \%)$ & \\
\hline
\end{tabular}

CVP, central venous port; GI, gastrointestinal tract

medical charts and imaging findings was conducted at a single center.

\section{Patients}

The study cohort included 254 consecutive patients who underwent CVP placement (right, 134; left, 120). Patients who were diagnosed with malignant tumor(s), and were $\geq$ 20 years of age, and underwent image-guided CVP placement between January and August 2012, were included. Patients who underwent catheterization with exchange from a previously inserted catheter were excluded. Up to 2011, the left side was chosen as a department rule at the authors' institution unless the patient exhibited any problems on the right side. After 2011, the side of placement was chosen according to operator preference, patient choice, and history of previous surgery on the chest wall and neck. Patient characteristics are summarized in Table 1. This study complied with the Health Insurance Portability and Accountability Act (HIPAA); the requirement for approval from the institutional review board was waived. Informed written consent for the procedures was obtained from all patients.

\section{CVP placement}

All CVP placements were performed under local anesthesia. SCV puncture was performed using real-time, long-axis ultrasound guidance and a CVP kit (Orca, Sumitomo Bakelite Co., Tokyo, Japan). After insertion of an 18-gauge needle into the SCV, a 0.032-inch guide wire was advanced under fluoroscopy and a 5 Fr indwelling catheter was advanced, also under fluoroscopic guidance. The position of the catheter tip was adjusted around the cavo-atrial junction. A port was implanted under the skin of the anterior chest wall and sutured using 3-0 nylon threads. The holes on the periphery of the port were fixed to the fascia or subcutaneous adipose with thread. Six board-certified interventional radiologists and 7 residents performed the procedures during the study period. Procedures performed by residents were supervised by certified interventional radiologists.

\section{Outcome measures}

The primary outcome measure was the rate of AEs in the right- and left-CVP groups. Secondary outcome measures included technical success, AE profile, and imaging findings on contrast-enhanced computed tomography (CE-CT).

\section{Statistical analysis}

Demographic information and baseline variables were summarized using descriptive statistics. Categorical variables were compared using the chi-squared test, and continuous variables were compared using the Student' s t test; the statistical significance level was set at .05. All statistical analyses were performed using SPSS version 20 (IBM Corporation, Armonk, NY, USA).

\section{Results}

\section{Technical results}

Technical success was achieved in $133(99 \%)$ and 119 $(99 \%)$ patients in the right- and left-CVP groups, respectively $(P=1.000)$. SCV access failed in one patient in the right- and one in the left-CVP group; consequently, the internal jugular vein was used for venous access. The median procedural time was $30 \mathrm{~min}$ in both groups $(P=.076)$.

\section{AEs}

No severe AEs occurred during the CVP placement procedure. The rates of intraprocedural AEs (Table 2) were not significantly different between the groups. During the follow-up time of 347 days in right- and 530 days in leftCVP groups $(P=.274)$, the rate of catheter occlusion was significantly higher in the left-CVP group (right $0 \%$; left 
Table 2. Adverse Events

\begin{tabular}{|c|c|c|c|}
\hline Adverse event (AE) & $\begin{array}{l}\text { Right } \\
(\mathrm{N}=134)\end{array}$ & $\begin{array}{l}\text { Left } \\
(\mathrm{N}=120)\end{array}$ & $P$ \\
\hline \multicolumn{4}{|l|}{ Intraprocedural AE } \\
\hline Pneumothorax & $1(0.7 \%)$ & $1(0.8 \%)$ & 1.000 \\
\hline Hematoma & $3(2.2 \%)$ & $2(1.7 \%)$ & .624 \\
\hline Air embolism & 0 & $2(1.7 \%)$ & .222 \\
\hline \multicolumn{4}{|l|}{ Postprocedural AE } \\
\hline CVP-related infection & $8(6.0 \%)$ & $5(4.2 \%)$ & .579 \\
\hline Injection resistance & $2(1.5 \%)$ & $7(5.8 \%)$ & .099 \\
\hline Catheter occlusion & 0 & $7(5.8 \%)$ & .005 \\
\hline Fibrin sheath & $1(0.7 \%)$ & $4(3.3 \%)$ & .192 \\
\hline $\begin{array}{l}\text { Symptomatic venous } \\
\text { thrombosis }\end{array}$ & $1(0.7 \%)$ & $5(4.2 \%)$ & .103 \\
\hline Removal of CVP system due to AEs & $7(5.2 \%)$ & $12(10 \%)$ & .161 \\
\hline
\end{tabular}

CVP, central venous port
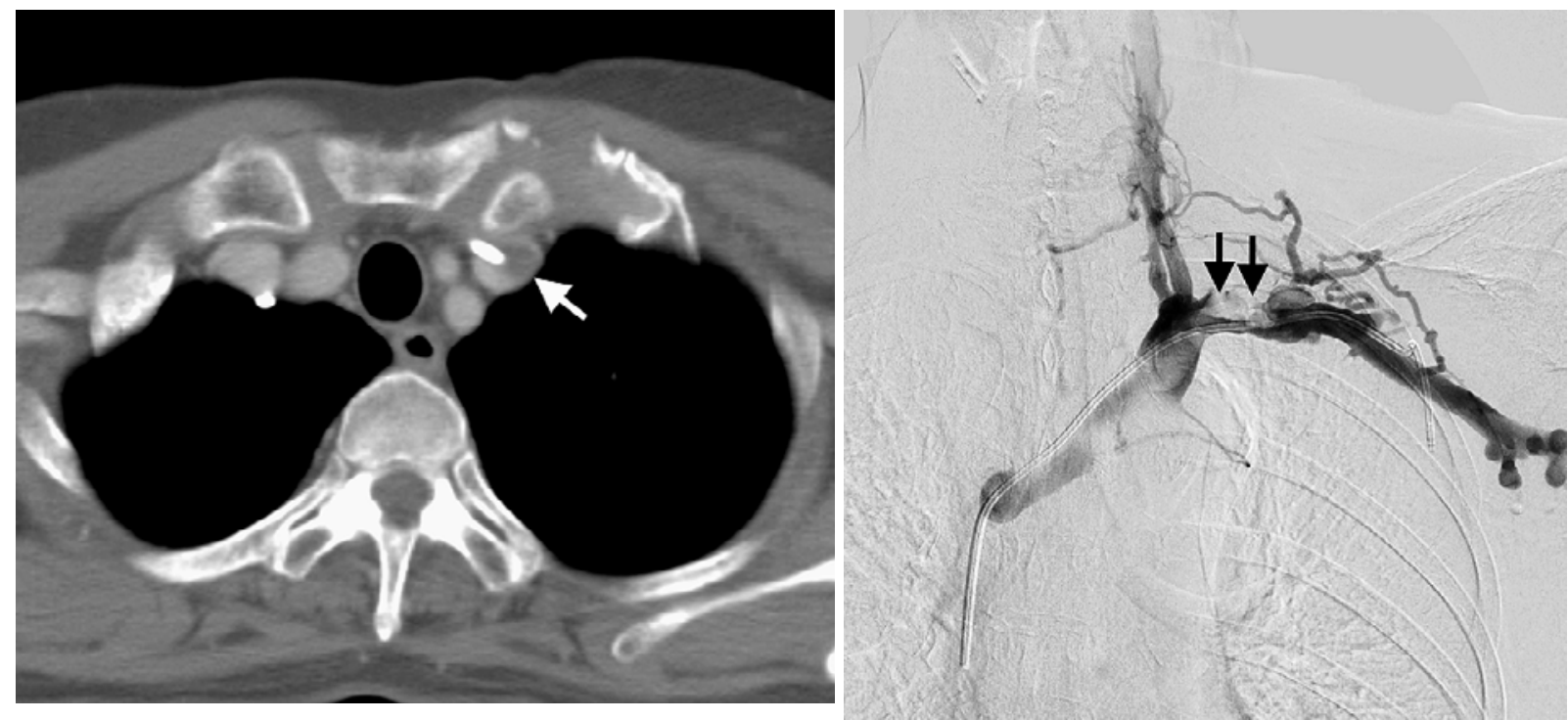

Fig. 1.

a. Contrast-enhanced computed tomography (CE-CT) demonstrating thrombus formation around the catheter in the left subclavian vein.

b. Venography performed on the same day as CE-CT demonstrates a filling defect in left subclavian vein, consistent with thrombus.

5.8\%, $P=.005$ ) (Table 2). No significant differences were observed for other postprocedural AEs or the rate of removal of CVPs due to AEs. The rate of injection resistance tended to be higher in the left-CVP $(5.8 \%)$ than in the rightCVP $(1.5 \%)$ group, although there was no statistically significant difference $(P=.099)$.

\section{CE-CT findings (Fig. 1)}

CE-CT was performed for disease follow-up after CVP placement in 62 patients $(46.2 \%)$ in the right- and 87 $(72.5 \%)$ in the left-CVP groups. The rate of venous thrombosis in the left-CVP group (19/87 [21.8\%]) was significantly higher than in the right-CVP group (3/62 [4.8\%]) $(P$
$<.001)$. Thrombus was evident around the indwelling catheter in all patients. A summary of venous thrombosis on CECT is shown in Table 3 .

\section{Discussion}

The present study compared AEs and imaging findings after CVP placement in the right or left SCV. Analysis revealed no differences between the two groups in terms of technical success and intraprocedural AEs. In contrast, leftCVP was associated with a significantly higher rate of postprocedural AEs involving catheter occlusion (right, 0\%; left, $5.8 \%, P=.005$ ). Both injection resistance (right, $1.5 \%$; left, 
Interventional Radiology 2018; 3: 121-125

Table 3. Venous Thrombosis on CE-CT

\begin{tabular}{llll}
\hline CE-CT performed & $\begin{array}{l}\text { Right } \\
62\end{array}$ & $\begin{array}{l}\text { Left } \\
87\end{array}$ & $P$ \\
\hline Venous thrombosis & $3(4.8 \%)$ & $19(21.8 \%)$ & $<.001$ \\
\hline Location of thrombus & & & \\
SCV only & 1 & 2 & \\
SVC only & 2 & 9 & .178 \\
SCV and SVC & 0 & 8 & \\
Time to presentation of venous & 51.0 & 95.5 & \\
thrombosis on CE-CT (days) & & & \\
Symptomatic venous thrombosis & 1 & 3 & \\
\hline
\end{tabular}

CE-CT, contrast-enhanced computed tomography; SCV, subclavian vein;

SVC, superior vena cava

$5.8 \%, P=.099)$ and image-confirmed venous thrombosis (right, 4.8\%; left, $21.8 \%, P<.001$ ) occurred more frequently in the left-CVP group. The rates of CVP-related infection were virtually equivalent in the two groups (right, 6.0\%; left, $4.2 \%$ ). These findings are important because maintaining use of a CVP may facilitate treatments such as intravenous chemotherapy and palliation of dehydration.

There is a paucity of comparative studies investigating right versus left placement of a CVP. A retrospective, singlecenter study by Tsai et al. reported lower rates of failure of SCV puncture, surgical complications, and catheter occlusion in the right-CVP group $(\mathrm{n}=1029)$ than in the left-CVP group ( $n=819)$ in patients requiring chemotherapy [3]. These findings are in agreement with the rate of catheter occlusion in the present study; however, technical success and intraprocedural complications in our study did not differ between the two groups. A possible explanation is the use of imaging guidance in our study in contrast with the landmark technique used in the study by Tsai et al.[3] The utility of ultrasound guidance to reduce AEs and failed catheterization has been reported $[4,5]$. Similarly, the high rate of technical success and low rates of intraprocedural AEs were achieved in the current study despite the involvement of various operators, including residents, presumably due to the use of image guidance with ultrasound and fluoroscopy.

Regarding postprocedural AEs, left-CVP was associated with higher rates of catheter occlusion and injection resistance in the present study, similar to the study by Tsai et al.[3]. Previous studies investigating hemodialysis blood access also reported a higher incidence of catheter malfunction with left internal jugular access versus right internal jugular access $[6,7]$. In a study by Wivell et al., catheter survival was essentially the same in right and left access at 30 days (right, 91\%; left, 85\%); however, left-access resulted in a significant decrease in catheter survival at 60 days (right, $83 \%$; left, 61\%) and 6 months (right, 74\%; left, 45\%). The similarity in the results of SCV access in the present study and internal jugular access in previous studies may be associated with anatomical differences in more central, right- and left-brachiocephalic veins. An analysis of the threedimensional anatomy of the left central veins using CT revealed that the angle between the left brachiocephalic vein and superior vena cava was $116( \pm 7)$ degrees, and the angulation of the left brachiocephalic vein as it traverses the aorta and left brachiocephalic artery was $106( \pm 9)$ degrees, while the right brachiocephalic trunk provides a direct vertical path to the superior vena cava [1]. The authors, Salik et al., postulated that a catheter traversing these angulated segments may irritate the vessel endothelium and be subjected to extrinsic compression and repetitive mechanical trauma from the pulsation of the aorta and the brachiocephalic artery and, thus, potentially contribute to stenosis and occlusion of the vein.

Cancer patients have a 4- to 6-fold risk for developing thrombotic events compared with the non-cancer population [8]. The reported incidence of venous thrombosis associated with $\mathrm{CV}$ catheters in oncological patients is high and variable $(12.0 \%$ to $66.0 \%)[9,10]$. On the other hand, the incidence of venous thrombosis associated with CVP in oncological patients $(0.2$ to $1.7 \%)$ was lower than data from CV catheters [3, 5, 10-15]. A possible explanation for the difference is that most CVPs are used in ambulatory settings while CV catheters are used in hospitalized patients because they are predisposed to risk for venous thrombosis (i.e., active cancer, or comorbid conditions such as infection and immobility). Nevertheless, the influence of the side of catheter placement has not been fully addressed.

Guidelines from the American Society of Clinical Oncology (ASCO) recommend venous thromboembolism prophylaxis with anticoagulation for most hospitalized patients with cancer [16]. For oncological patients with symptomatic deep vein thrombosis (DVT) and pulmonary embolism (PE), lowmolecular weight heparin is recommended for initial and long-term secondary prophylaxis [16]. Importantly, treatment of incidental DVT and PE in the same manner as symptomatic treatment was recommended in the ASCO guideline based on consensus [16]. Thus, our results revealing higher rates of venous thrombosis on follow-up CE-CT in the left- 
CVP group may have an important implication for the choice of the side of CVP placement.

Several limitations of this study should be acknowledged. First, confounding factors due to sample bias could not be eliminated due to the retrospective study design. Second, the use and the type of anticancer treatments were not evaluated. The purpose of CVP placement differed significantly among patients, and the higher rate of chemotherapy in the left CVP group would be associated with a higher rate of use of chemotherapeutic- and molecular-targeted agents that are known risk factors for venous thrombosis. Third, the relatively small population limited comparisons between the two groups.

In conclusion, higher rates of postoperative AEs and image-confirmed venous thrombosis were observed in the left-side approach in the placement of CVP. Re-evaluation may be required for left-CVP in oncological patients.

Conflict of interest: The authors have no conflicts of interest to declare.

Acknowledgement: This research was supported by the Practical Research for Innovative Cancer Control (16ck0106058h0003) from the Japan Agency for Medical Research and Development (AMED), Health and Labor Sciences Research Grant (H26-055) from the Ministry of Health, Labour and Welfare of Japan, and the National Cancer Center Research and Development Fund (26-A-27).

\section{References}

1. Salik E, Daftary A, Tal MG. Three-dimensional anatomy of the left central veins: implications for dialysis catheter placement. J Vasc Interv Radiol. 2007; 18(3):361-364.

2. Sulek CA, Blas ML, Lobato EB. A randomized study of left versus right internal jugular vein cannulation in adults. J Clin Anesth. 2000; 12(2):142-145.

3. Tsai YF, Ku YH, Chen SW, Huang WT, Lu CC, Tsao CJ. Rightand left-subclavian vein port-a-cath systems: comparison of complications. Eur Surg Res. 2012; 49(2):66-72.

4. Lalu MM, Fayad A, Ahmed O, Bryson GL, Fergusson DA, Barron $\mathrm{CC}$, et al. Ultrasound-guided subclavian vein catheterization: a systematic review and meta-analysis. Crit Care Med. 2015; 43(7): 1498-1507.

5. Sakamoto N, Arai Y, Takeuchi Y, Takahashi M, Tsurusaki M, Sugimura K. Ultrasound-guided radiological placement of central venous port via the subclavian vein: a retrospective analysis of
500 cases at a single institute. Cardiovasc Intervent Radiol. 2010; 33(5):989-994.

6. Salgado OJ, Urdaneta B, Colmenares B, Garcia R, Flores C. Right versus left internal jugular vein catheterization for hemodialysis: complications and impact on ipsilateral access creation. Artif Organs. 2004; 28(8):728-733.

7. Wivell W, Bettmann MA, Baxter B, Langdon DR, Remilliard B, Chobanian M. Outcomes and performance of the Tesio twin catheter system placed for hemodialysis access. Radiology. 2001; 221 (3):697-703.

8. Timp JF, Braekkan SK, Versteeg HH, Cannegieter SC. Epidemiology of cancer-associated venous thrombosis. Blood. 2013; 122 (10):1712-1723.

9. Agnelli G, Verso M. Therapy Insight: venous-catheter-related thrombosis in cancer patients. Nat Clin Pract Oncol. 2006; 3(4): 214-222.

10. Vescia S, Baumgartner AK, Jacobs VR, Kiechle-Bahat M, Rody A, Loibl S, et al. Management of venous port systems in oncology: a review of current evidence. Ann Oncol. 2008; 19(1):9-15.

11. Akahane A, Sone M, Ehara S, Kato $K$, Tanaka R, Nakasato T. Subclavian vein versus arm vein for totally implantable central venous port for patients with head and neck cancer: a retrospective comparative analysis. Cardiovasc Intervent Radiol. 2011; 34(6): 1222-1229.

12. Inaba $Y$, Yamaura H, Sato $Y$, Najima M, Shimamoto H, Nishiofuku $\mathrm{H}$, et al. Central venous access port-related complications in outpatient chemotherapy for colorectal cancer. Jpn J Clin Oncol. 2007; 37(12):951-954.

13. Biffi R, de Braud F, Orsi F, Pozzi S, Mauri S, Goldhirsch A, et al. Totally implantable central venous access ports for long-term chemotherapy. A prospective study analyzing complications and costs of 333 devices with a minimum follow-up of 180 days. Ann Oncol 1998; 9(7):767-773.

14. Cil BE, Canyigit M, Peynircioglu B, Hazirolan T, Carkaci S, Cekirge S, et al. Subcutaneous venous port implantation in adult patients: a single center experience. Diagn Interv Radiol 2006; 12 (2):93-98.

15. Yildizeli B, Lacin T, Batirel HF, Yuksel M. Complications and management of long-term central venous access catheters and ports. J Vasc Access 2004; 5(4):174-178.

16. Lyman GH, Khorana AA, Kuderer NM, Lee AY, Arcelus JI, Balaban EP, et al. Venous thromboembolism prophylaxis and treatment in patients with cancer: American Society of Clinical Oncology clinical practice guideline update. J Clin Oncol. 2013; 31(17): 2189-2204. 